\title{
Search for heavy neutrinos at the NA48/2 and NA62 experiments at CERN
}

\author{
Peruzzo Letizia ${ }^{1, a}$ on behalf of the NA48/2 and NA62 Collaborations ${ }^{b}$ \\ ${ }^{1}$ Johannes Gutenberg University, Mainz (Germany)
}

\begin{abstract}
The NA48/2 experiment at CERN has collected large samples of charged kaons decaying into a pion and two muons for the search of heavy nuetrinos. In addition, its successor NA62 has set new limits on the rate of charged kaon decay into a heavy neutral lepton (HNL) and a lepton, with $\ell=e, \mu$, using the data collected in 2007 and 2015. New limits on heavy neutrinos from kaon decays into pions, muons and positrons are presented in this report.
\end{abstract}

\section{Introduction}

The massive nature of neutrinos has unambiguously been demonstrated by the observation of neutrino oscillations, although, within the Standard Model (SM), neutrinos are massless and right-handed neutrino states are not included. A lot of SM extensions try to accomodate the neutrino mass in a way consistent with experimental observation by including heavy neutral leptons (HNLs), which can mix with ordinary SM particles. The possibility to include right-handed neutrinos is provided, for example, by the Neutrino Minimal Standard Model ( $v$ MSM) [1]: a SM extension in which 18

\footnotetext{
ae-mail: lperuzzo@uni-mainz.de

$\mathrm{b}_{\text {for the NA48/2 and NA62 Collaborations: }}$

R. Aliberti, F. Ambrosino, R. Ammendola, B. Angelucci, A. Antonelli, G. Anzivino, R. Arcidiacono, M. Barbanera, A. Biagioni, L. Bician, C. Biino, A. Bizzeti, T. Blazek, B. Bloch-Devaux, V. Bonaiuto, M. Boretto, M. Bragadireanu, D. Britton, F. Brizioli, M.B. Brunetti, D. Bryman, F. Bucci, T. Capussela, A. Ceccucci, P. Cenci, V. Cerny, C. Cerri, B. Checcucci, A. Conovaloff, P. Cooper, E. Cortina Gil, M. Corvino, F. Costantini, A. Cotta Ramusino, D. Coward, G. D’Agostini, J. Dainton, P. Dalpiaz, H. Danielsson, N. De Simone, D. Di Filippo, L. Di Lella, N. Doble, B. Dobrich, F. Duval, V. Duk, J. Engelfried, T. Enik, N. Estrada-Tristan, V. Falaleev, R. Fantechi, V. Fascianelli, L. Federici, S. Fedotov, A. Filippi, M. Fiorini, J. Fry, J. Fu, A. Fucci, L. Fulton, E. Gamberini, L. Gatignon, G. Georgiev, S. Ghinescu, A. Gianoli, M. Giorgi, S. Giudici, F. Gonnella, E. Goudzovski, C. Graham, R. Guida, E. Gushchin, F. Hahn, H. Heath, T. Husek, O. Hutanu, D. Hutchcroft, L. Iacobuzio, E. Iacopini, E. Imbergamo, B. Jenninger, K. Kampf, V. Kekelidze, S. Kholodenko, G. Khoriauli, A. Khotyantsev, A. Kleimenova, A. Korotkova, M. Koval, V. Kozhuharov, Z. Kucerova, Y. Kudenko, J. Kunze, V. Kurochka, V. Kurshetsov, G. Lanfranchi, G. Lamanna, G. Latino, P. Laycock, C. Lazzeroni, M. Lenti, G. Lehmann Miotto, E. Leonardi, P. Lichard, L. Litov, R. Lollini, D. Lomidze, A. Lonardo, P. Lubrano, M. Lupi, N. Lurkin, D. Madigozhin, I. Mannelli, G. Mannocchi, A. Mapelli, F. Marchetto, R. Marchevski, S. Martellotti, P. Massarotti, K. Massri, E. Maurice, M. Medvedeva, A. Mefodev, E. Menichetti, E. Migliore, E. Minucci, M. Mirra, M. Misheva, N. Molokanova, M. Moulson, S. Movchan, M. Napolitano, I. Neri, F. Newson, A. Norton, M. Noy, T. Numao, V. Obraztsov, A. Ostankov, S. Padolski, R. Page, V. Palladino, C. Parkinson, E. Pedreschi, M. Pepe, M. Perrin-Terrin, L. Peruzzo, P. Petrov, F. Petrucci, R. Piandani, M. Piccini, J. Pinzino, I. Polenkevich, L. Pontisso, Yu. Potrebenikov, D. Protopopescu, M. Raggi, A. Romano, P. Rubin, G. Ruggiero, V. Ryjov, A. Salamon, C. Santoni, G. Saracino, F. Sargeni, V. Semenov, A. Sergi, A. Shaikhiev, S. Shkarovskiy, D. Soldi, V. Sougonyaev, M. Sozzi, T. Spadaro, F. Spinella, A. Sturgess, J. Swallow, S. Trilov, P. Valente, B. Velghe, S. Venditti, P. Vicini, R. Volpe, M. Vormstein, H. Wahl, R. Wanke, B. Wrona, O. Yushchenko, M. Zamkovsky, A. Zinchenko.
} 
new parameters in the Lagrangian explain the open questions about the existence of Dark Matter and the baryon asymmetry of the universe consistent with the observation of neutrino oscillation. In the $v$ MSM three massive right-handed neutrinos are introduced to the SM particle budget. The lightest among the three massive neutrinos, with mass of $O\left(10 \mathrm{keV} / \mathrm{c}^{2}\right)$, is considered a dark matter candidate while the other two, with masses of $O\left(100 \mathrm{MeV} / c^{2}\right)$ give masses to the SM neutrinos via the see-saw mechanism and introduce extra $\mathrm{CP}$ violating phases to explain the baryon asymmetry. Since the HNL masses are chosen to be at or below the scale of the electroweak interaction one of the most promising sectors to study HNLs are kaon decays.

Large samples of kaon decays have been collected during the last 20 years by the fixed-target experiments NA48 and NA62, located at the Super Proton Synchrotron (SPS) at CERN.

This report presents three different searches for HNLs using data collected by the NA48/2 experiment in 2003-2004 and by the NA62 experiment in 2007 and 2015.

\section{Heavy Neutrino searches}

Two different strategies can in principle be used in HNL searches, depending on the assumption made on HNL properties, such as mass and lifetime.

\section{HNL production}

The first approach searches for HNL in pions and kaons decays looking for peaks in the missing mass spectra of two-body decays. Peak searches in the kaon sector come from two-body decays like $K \rightarrow \ell N$ with $\ell=e, \mu$ and $N$ the HNL. The branching ratio of these decays depends on the mass of the HNL and on the element $U_{\ell 4}$ of the matrix that mixes HNLs with leptons, and can be expressed as [2]:

$$
\mathcal{B R}(K \rightarrow \ell N)=\mathcal{B R}\left(K \rightarrow \ell v_{\ell}\right) \cdot \rho_{\ell}\left(m_{N}\right) \cdot\left|U_{\ell 4}\right|^{2}
$$

where $\mathcal{B R}\left(K \rightarrow \ell v_{\ell}\right)$ is the branching ratio of the decay into a lepton and a SM neutrino and $\rho_{\ell}\left(m_{N}\right)$ is a kinematic factor, which depends on the HNL mass and takes into account the phase space and the helicity suppression. For most of the allowed range the product $\mathcal{B R}\left(K \rightarrow \ell v_{\ell}\right) \cdot \rho_{\ell}\left(m_{N}\right)$ is $O(1)$.

The existing peak search limits on HNLs come from experiments using stopped kaons. In 2007 the NA62 experiment at CERN was the first experiment to perform an HNL search using kaon decays in-flight. It continued this search in 2015 with a complete new apparatus. This report presents the search of HNLs in $K^{+} \rightarrow \mu^{+} N$ and $K^{+} \rightarrow e^{+} N$ decays using data collected by the NA62 experiment in 2007 and 2015, respectively.

\section{HNL decay}

The second approach looks for HNL decay products. In the $v$ MSM framework, HNLs decay via both charged and neutral currents into SM particles (such as neutrinos, pions and leptons) with a branching ratio proportional to the HNL mass and the mixing matrix element $\left|U_{\ell 4}\right|^{2}$ :

$$
\mathcal{B R} \sim\left|U_{\ell 4}\right|^{2} \cdot m_{N}^{3}
$$

Usually decay searches are performed in beam dump experiments where HNLs are produced from mesons decays. However, decay searches can also be studied in lepton number violating decays (LNV) with $\Delta L=2$ where an on-shell or virtual Majorana neutrino is produced. Limits from decay searches (both in beam dump experiments and in LNV decays) are stronger than those from peak searches, but less robust since they depend on assumptions on the HNL decays. The large sample of 
kaon decays with multiple charged particles in the final state, collected in 2003-2004 by the NA48/2 experiment, allows to search for the forbidden LNV decay $K^{ \pm} \rightarrow \pi^{\mp} \mu^{ \pm} \mu^{ \pm}$as well as for two-body resonances in $K^{ \pm} \rightarrow \pi \mu \mu$ decays, also presented in this report.

\section{Kaon experiments at CERN}

The NA62 experiment is the latest in a series of fixed-target experiments located in the north area of the CERN Super Proton Synchrotron (SPS) facility, using the decay-in-flight technique to explore kaon decays. The tradition has begun in 1979 with the NA31 experiment which aimed at studying CP violation in neutral kaon decays [3]. In 1990 the experiment NA48 was proposed to measure $\mathfrak{R}\left(\epsilon^{\prime} / \epsilon\right)$ with high accuracy [4]. In 2003-2004 the attention moved from CP violation in neutral kaons to CP violation in charged kaon decays in the NA48/2 experiment. In 2006 the successor NA62 was proposed to measure ultra rare kaon decays [5]. In its first phase in 2007-2008, called NA62 ${ }_{R K}$ phase, the experiment benefited of the beam and detector of NA48/2 in order to measure the ratio $R_{K}=\Gamma\left(K^{ \pm} \rightarrow e^{ \pm} v\right) / \Gamma\left(K \rightarrow \mu^{ \pm} v\right)[6]$. NA62 with the complete new apparatus has started to take data in 2015 [7]. The main goal of the experiment is to precisely measure the very rare decay $K^{+} \rightarrow \pi^{+} v \bar{v}$ $\left(\mathcal{B R}_{S M}=(8.4 \pm 1.0) \times 10^{-11}[8]\right)$ by collecting around 100 events.

\subsection{NA48/2 in 2003-2004}

The NA48/2 experiment was located in the north area of the CERN SPS and was a successor of the NA48 experiment with almost the same detector setup. The primary goal of NA48/2 was the search for direct CP violation in $K^{ \pm} \rightarrow \pi^{ \pm} \pi^{+} \pi^{-}$and $K^{ \pm} \rightarrow \pi^{ \pm} \pi^{0} \pi^{0}$ decays [9] with about 100 days of effective data taking in 2003-2004. The experiment used $400 \mathrm{GeV} / c$ protons provided by the SPS which, hitting on a Beryllium target, produced a secondary hadronic beam. Thanks to a system of magnets and collimators only charged particles with momenta of $(60 \pm 3) \mathrm{GeV} / c$ were selected and aligned with the longitudinal axis of the detector within $1 \mathrm{~mm}$ and with a transverse size of about $1 \mathrm{~cm}$. The experiment was recording the decays of $\mathrm{K}^{+}$and $\mathrm{K}^{-}$mesons inside the fiducial decay region located in a $114 \mathrm{~m}$ long cylindrical vacuum tank. After the vacuum tank a thin Kevlar window separated the vacuum from a helium vessel at atmospheric pressure in which a magnetic spectrometer was located. The spectrometer consisted of four drift chambers $(\mathrm{DCH})$ and a dipole magnet, placed between the second and the third chamber, which provided a horizontal momentum kick of $p_{t}=120 \mathrm{MeV} / c$ for charged particles. Each chamber had four different views and a spatial resolution of $\sigma_{x, y}=90 \mu \mathrm{m}$. The nominal momentum resolution of the spectrometer was $\sigma_{p} / p=(1.02 \oplus 0.044 p) \%$ where $p$ is given in $\mathrm{GeV} / c$. A scintillating hodoscope (HOD) followed the spectrometer. It was divided in four quadrants by a horizontal and a vertical plane of strip-shaped counters. The HOD was used in the trigger chain to provide a fast time measurement for charged particles with a 150 ps time resolution. A quasi homogeneous electromagnetic calorimeter filled with liquid krypton ( $\mathrm{LKr}$ ) and a depth of $27 X_{0}$ was used both for photon detection and particle identification. It had an energy resolution of $\sigma_{E} / E=0.032 / \sqrt{E} \oplus 0.09 / E \oplus 0.0042(E$ in $\mathrm{GeV})$ corresponding to $\sigma_{E} / E=0.94 \%$ at $20 \mathrm{GeV}$. The particle identification was completed by a hadronic calorimeter and a muon veto system (MUV). The hadronic calorimeter consisted of alternated iron and scintillator planes, while the MUV of three plastic scintillator strips planes each of them preceded by a $80 \mathrm{~cm}$ iron wall. A more detailed description of the experiment can be found in [4].

\subsection{NA62 ${ }_{R K}$ phase in 2007-2008}

The NA62 experiment started its data taking in 2007 with the measurement of the $R_{K}$ ratio [6]. In this first phase it used the slightly modified beam line and detector of the previous experiment NA48/2. 
The kaons beams (both positive and negative) were centered at $74 \mathrm{GeV} / c$ (instead of $60 \mathrm{GeV} / c$ ) with a spread of $\pm 1.4 \mathrm{GeV} / c$. The beam intensity was reduced by a factor of about 10 with respect to NA48/2 in order to enable a minimum-bias trigger configuration with high efficiency and to minimize the accidental background.

\subsection{NA62 in 2015}

A complete new apparatus was developed after the first NA62 $R K$ phase in order to reach the main goal of the experiment: measure the very rare decay $K^{+} \rightarrow \pi^{+} v \bar{v}$ with $10 \%$ precision on the branching ratio. The construction finished in 2015 and after the full commissioning in September 2016 the experiment started the physics data taking. The experiment uses the SPS proton beam $(400 \mathrm{GeV} / \mathrm{c})$, which hitting on a Beryllium target produce a secondary hadronic beam. The kaons are only $6 \%$ of the total particles. The beam has a momentum of $(75 \pm 1) \mathrm{GeV} / c$ and a nominal intensity of $750 \mathrm{MHz}$. The detector apparatus extends over $270 \mathrm{~m}$ from the target to the beam dump located at the end of the experiment. The possibility to tag the kaon and measure times and momenta of the beam particles represents one of the main improvements with respect to the 2007 setup. The kaon tagging and the precise timing measurement of the beam particles are provided by a Cherenkov detector (KTAG). It is followed by a beam spectrometer (Gigatracker or GTK), three silicon pixel stations exposed to the full beam rate of $750 \mathrm{MHz}$. In order to improve the detection of the kaon decays the four drift chambers were replaced by a magnetic spectrometer of four chambers with STRAW tubes placed in a vacuum. The particle identification is performed by the Ring Imaging Cherenkov (RICH) detector, an electromagnetic and a hadronic calorimeter and a muon veto (MUV) system. A hermetic photon system covering an angle up to $50 \mathrm{mrad}$ completes the detector layout. A detail description of the NA62 beam line and detector can be found in [7].

The HNL search presented in this report used the data collected in 5 days of a pilot run in 2015 at a beam intensity varying from $0.4 \%$ to $1.3 \%$ of the nominal intensity, in which the GTK was not present.

\section{HNL search in $K^{ \pm} \rightarrow \pi \mu \mu$ decays}

Two different samples were selected on the data collected in 2003-2004, based on the sign of the muons in the final state: candidates from the lepton number violating decay $K^{ \pm} \rightarrow \pi^{\mp} \mu^{ \pm} \mu^{ \pm}\left(K_{\pi \mu \mu}^{L N V}\right)$ where the muons have the same sign and candidates from the lepton number conserving decay, with two opposite sign muons, $K^{ \pm} \rightarrow \pi^{ \pm} \mu^{+} \mu^{-}\left(K_{\pi \mu \mu}^{L N C}\right)$. Upper limits were set on the branching ratio of the LNV decay, as well as on the search for resonances in the invariant mass $\mathrm{M}_{\pi \mu}$ of the final state.

\subsection{Events selection}

Due to the decay vertex resolution, the signature given by a particle $X$ produced in $K \rightarrow \mu X$ and promptly decaying to $X \rightarrow \pi \mu$ is indistinguishable from a real three-track decay. Therefore the trigger logic of three-track events (the main trigger of the experiment) could be used.

The $K^{ \pm} \rightarrow \pi^{ \pm} \pi^{+} \pi^{-}\left(K_{3 \pi}\right)$ decay was used as a normalization channel: the same three-track topology and the small difference in mass between $\pi$ and $\mu$ allow a first order cancellation of systematic effects due to possible imperfect kaon beam description and detector and trigger inefficiencies.

The three-track events selection required a total charge of the three tracks equal to \pm 1 and a vertex inside the $98 \mathrm{~m}$ long fiducial decay region. For each track a momentum between 5 and $55 \mathrm{GeV} / c$ was required and a total momentum for the three tracks compatible with the beam momentum. A cut was 
applied to the total transverse momentum of the three tracks with respect to the beam direction, that is measured using the $K_{3 \pi}$ sample $\left(p_{t}<10 \mathrm{MeV} / c\right)$. The three tracks forming the vertex had to be consistent in time with the trigger and within $10 \mathrm{~ns}$ from the average time of the three tracks. The three tracks had to consist of a $\pi^{ \pm}$candidate and two muons with the same/opposite sign for $K_{\pi \mu \mu}^{L N V}$ and $K_{\pi \mu \mu}^{L N C}$, respectively. Muon and pion particle identification was done by using hits in the MUV and the ratio of the energy deposited in the $\mathrm{LKr}$ and the momentum measured by the $\mathrm{DCH}$.

The signal region satisfies $\left|M_{\pi \mu \mu}-M_{K}\right|<5(8) \mathrm{MeV} / c^{2}$ where $M_{\pi \mu \mu}$ is the invariant mass of the three tracks in the $\pi^{\mp} \mu^{ \pm} \mu^{ \pm}\left(\pi^{ \pm} \mu^{+} \mu^{-}\right)$hypothesis and $M_{K}$ is the nominal $K^{ \pm}$mass. The selected region corresponds to \pm 2 and \pm 3.2 times the resolution of $M_{\pi \mu \mu}$, respectively, for $K_{\pi \mu \mu}^{L N V}$ and $K_{\pi \mu \mu}^{L N C}$, where $\sigma_{M(\pi \mu \mu)}=2.5 \mathrm{MeV} / c^{2}$. The different cuts on the mass resolution are due to the different background composition in the two samples. The data/Monte Carlo agreement was studied in the control region $456 \mathrm{MeV} / c^{2}<M_{\pi \mu \mu}<480 \mathrm{MeV} / c^{2}$.

For the $K_{3 \pi}$ normalization channel the pion identification criteria used for the signal were applied only for the track with opposite electric charge with respect to the kaon. The invariant mass of the three pions had to satisfy $\left|M_{3 \pi}-M_{K}\right|<5 \mathrm{MeV} / c^{2}$ corresponding to $\pm 3 \sigma_{3 \pi}\left( \pm 5.1 \mathrm{MeV} / c^{2}\right)$. The number of $K^{ \pm}$decays in the fiducial region was measured to $N_{K}=1.637 \times 10^{11}$ using the normalization channel $K_{3 \pi}$ with $N_{3 \pi}=1.367 \times 10^{7}$ and the acceptance of $A_{3 \pi}=14.96 \%$.

The events passing the signal selection are shown in figure 1 for both data and Monte Carlo simu-
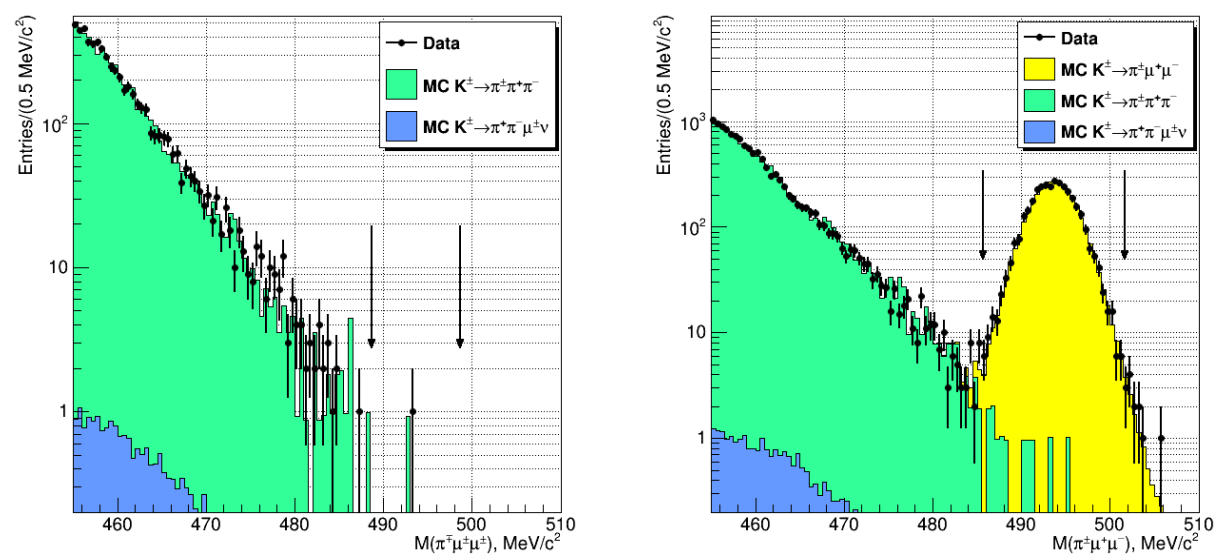

Figure 1. $M_{\pi \mu \mu}$ mass distribution for data and Monte Carlo simulation for events that pass the $K_{\pi \mu \mu}^{L N V}$ (left) and the $K_{\pi \mu \mu}^{L N C}$ (right) selection.

lation. Only one event is observed in the signal region for the $K_{\pi \mu \mu}^{L N V}$ sample after applying the signal selection. The background expectation is $N_{b k g}=1.16 \pm 0.87_{\text {stat. }} \pm 0.12_{\text {syst. }}$, evaluated with Monte Carlo simulation and mostly composed of $K_{3 \pi}$ events in which two pions decay into muons. Since no signal was observed an upper limit was set on $\mathcal{B R}\left(K^{ \pm} \rightarrow \pi^{\mp} \mu^{ \pm} \mu^{ \pm}\right)$applying the Rolke-Lopez statistical method [10] to find the $90 \%$ confidence interval:

$$
\mathcal{B R}\left(K^{ \pm} \rightarrow \pi^{\mp} \mu^{ \pm} \mu^{ \pm}\right)=\frac{N_{\pi \mu \mu}^{L N V} \cdot A_{3 \pi}}{N_{3 \pi} \cdot D \cdot A\left(K_{\pi \mu \mu}^{L N V}\right)} \cdot \mathcal{B R}\left(K_{3 \pi}\right)<8.6 \times 10^{-11},
$$

where $D=100$ is the trigger downscaling, $N_{\pi \mu \mu}^{L N V}<2.92$ the upper limit on the signal events at $90 \%$ $\mathrm{CL}$ and $A\left(K_{\pi \mu \mu}^{L N V}\right)=(20.62 \pm 0.01) \%$ the signal acceptance evaluated with Monte Carlo simulation. 
In the $K_{\pi \mu \mu}^{L N C}$ selection a total of 3489 candidates were observed where the background contamination from $K_{3 \pi}$ was estimated to be $(0.36 \pm 0.10) \%$ using Monte Carlo simulation. This level of purity allows to consider $K_{\pi \mu \mu}^{L N C}$ as the only background for resonance searches in that sample. For a more complete description of the analysis refer to [11].

\subsection{Search for two-body resonances}

The search for the two-body resonances was performed on all events that survived $K^{ \pm} \rightarrow \mu^{ \pm} X$ with $X \rightarrow \pi \mu$ in the $K_{\pi \mu \mu}^{L N V}$ and $K_{\pi \mu \mu}^{L N C}$ selections respectively. In the scan of the invariant mass $M_{\pi \mu}$, the mass steps are given by $\sigma\left(M_{\pi \mu}\right) / 2$, where $\sigma\left(M_{\pi \mu}\right)$ is the mass resolution, and the signal window by $\pm 2 \sigma\left(M_{\pi \mu}\right)$ at each mass hypothesis. This means that the results obtained in neighbouring mass hypotheses are highly correlated, as the signal mass window is 8 times larger than the mass step.

In total, 284 and 267 mass hypotheses have been tested, covering the full kinematic range of the $M_{\pi \mu}$ distributions for the $K_{\pi \mu \mu}^{L N V}$ and $K_{\pi \mu \mu}^{L N C}$ candidates.

For the search of two-body resonances the Rolke-Lopez statistical method is used. The number of considered background events for the $K_{\pi \mu \mu}^{L N V}\left(K_{\pi \mu \mu}^{L N C}\right)$ candidates is $N=4(N=1)$. For each mass hypothesis of the resonances the local significance $z$ was evaluated as $z=\frac{N_{o b s}-N_{e x p}}{\sqrt{\delta N_{o b s}^{2}+\delta N_{\text {exp }}^{2}}}$, where $N_{o b s}$ and $N_{\text {exp }}$ are the number of observed and expected background events with their uncertainties $\delta N_{o b s}$ and $\delta N_{\text {exp }}$. Since the local significances never exceed 3 standard deviations, no signal was observed, and upper limits were set on the product $\mathcal{B R}\left(K^{ \pm} \rightarrow \mu X\right) \mathcal{B R}(X \rightarrow \pi \mu)$. The upper limits are calculated as a function of the resonance lifetime $\tau$ for each mass hypothesis $m_{i}$ by using the values of the signal acceptances (these also depend on the resonances mass and lifetime) and the upper limits on the number of signal events in each mass hypothesis:

$$
\left.\mathcal{B R}\left(K^{ \pm} \rightarrow \mu X\right) \mathcal{B R}(X \rightarrow \pi \mu)\right|_{m_{i}, \tau}=\frac{N_{s i g}^{i}}{N_{3 \pi} \cdot D} \cdot \frac{A_{3 \pi}}{A_{\pi \mu \mu}\left(m_{i}, \tau\right)} \cdot \mathcal{B R}\left(K_{3 \pi}\right)
$$

The obtained upper limits are shown in figure 2.
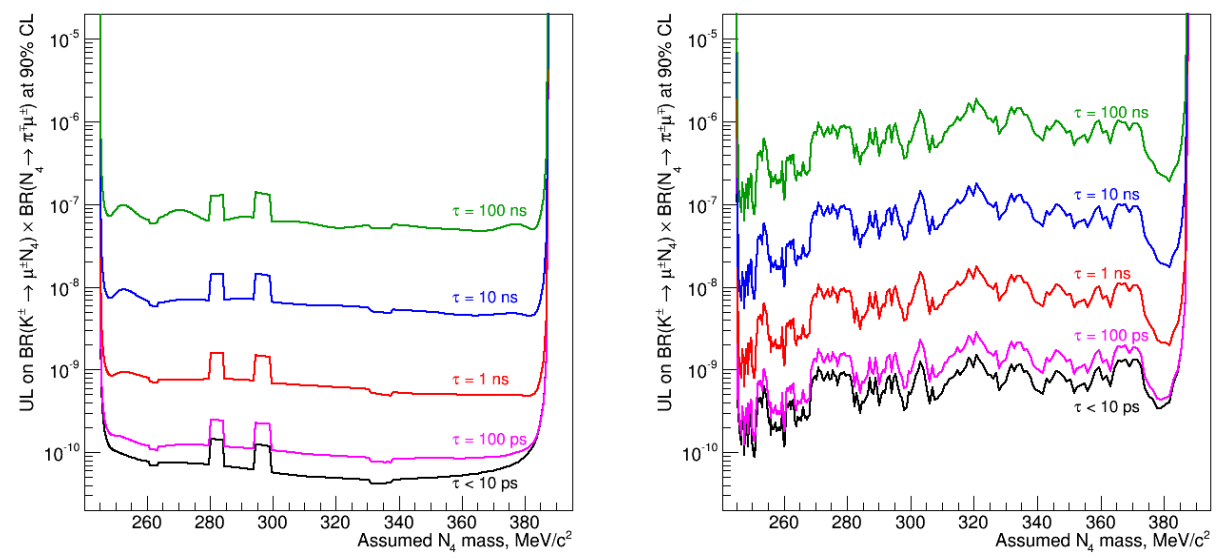

Figure 2. Obtained upper limits at $90 \% \mathrm{CL}$ on the products of branching ratios as functions of the resonance mass and lifetime: (left) $\mathcal{B R}\left(K^{ \pm} \rightarrow \mu^{ \pm} N_{4}\right) \mathcal{B R}\left(N_{4} \rightarrow \pi^{\mp} \mu^{ \pm}\right)$; (right) $\mathcal{B R}\left(K^{ \pm} \rightarrow \mu^{ \pm} N_{4}\right) \mathcal{B R}\left(N_{4} \rightarrow \pi^{ \pm} \mu^{\mp}\right)$; 


\section{HNL search in $K^{+} \rightarrow \ell^{+} N$ decay}

HNL production was studied in the $K^{+} \rightarrow \ell^{+} N$ decay, where $\ell=\mu, e$ and their decay in flight along the detector can be neglected. In fact, assuming $\left|U_{\ell 4}\right|^{2}<10^{-4}$ and considering HNL decays into SM particles, the mean free path of HNLs produced in NA62 is greater than $10 \mathrm{~km}$ for the mass ranges considered in the analysis.

\section{Muon channel}

The peak search for HNLs in the channel $K^{+} \rightarrow \mu^{+} N$ was performed using the data collected in 2007 from NA62 $2_{R K}$. Although both the $K^{+}$and $K^{-}$beams were available in this phase of the experiment only the positive component was taken into account in the analysis (about $43 \%$ of the total data) due to a higher muon halo rejection with respect to the negative component. A new mass range with respect to the previous analysis has been explored for the HNLs: $m_{N} \in[300,375] \mathrm{MeV} / \mathrm{c}^{2}$. For a more detailed description of the analysis refer to [12].

\section{Positron channel}

The peak search of HNLs in the channel $K^{+} \rightarrow e^{+} N$ was performed using the data collected in 2015 with the new NA62 detector layout. The beam spectrometer was not yet present and the kaon momenta and directions were evaluated by using the information of the reconstructed $K^{+} \rightarrow \pi^{+} \pi^{+} \pi^{-}$decay, in the same way as for the 2007 analysis. Upper limits were set for the HNL production in the mass range $170<m_{N}<448 \mathrm{MeV} / \mathrm{c}^{2}$.

\subsection{Events selection}

The $K^{+} \rightarrow \ell^{+} N$ decays required the presence of only one reconstructed track in the spectrometer and inside the acceptance of the so-called downstream detector. The track had to match with a beam particle, where its momentum and position were evaluated by fully reconstructed $K^{+} \rightarrow \pi^{+} \pi^{+} \pi^{-}$events. In 2015 the additional information from the KTAG allowed to identify the beam particle as a kaon. In the muon decay channel, muons were identified by requiring signal in the MUV associated in time and space with track. While in the positron channel, the electrons and positrons particle identification was done using the energy deposit in the LKr over the momentum in the spectrometer $(E / p)$, and the RICH information. Events with additional particle (except for the lepton) were rejected.

In both analysis the production of HNL was studied searching for peak in the missing mass spectrum: $m_{\text {miss. }}^{2}=\left(P_{K}-P_{\ell}\right)^{2}$, where $P_{\ell}$ represents the 4-momentum of the track reconstructed by the spectrometer under the mass hypothesis of $\ell$, and $P_{K}$ is the kaon momentum evaluated as the average of the momenta of the $3 \pi$ system in $K^{+} \rightarrow \pi^{+} \pi^{+} \pi^{-}$decays.

Figure 3 shows the squared missing mass spectrum for the events that pass the selection.

The HNL signal regions are defined as $m_{\text {miss. }} \in[300,375] \mathrm{MeV} / c^{2}$ and $m_{\text {miss. }} \in[170,448] \mathrm{MeV} / c^{2}$ for the 2007 and 2015 analysis, respectively. The number of kaons that decay in the fiducial regions was evaluated from the numbers of selected events with $m_{\text {miss. }}^{2}$ in the SM signal region and by the acceptance evaluated using MC simulation.

The obtained numbers of kaon decays are $N_{K}^{\mu}=(5.977 \pm 0.015) \times 10^{7}$ and $N_{K}^{e}=(3.01 \pm 0.011) \times 10^{8}$. Two background contributions were considered for 2007 analysis: muons coming from the beam (muon halo) which has been studied directly on the data using the control sample with the $K^{-}$beam, and background from decays evaluated with MC simulation. For the 2015 analysis the background was evaluated in the data looking in the side bands of the $m_{m i s s}^{2}$ distribution. 


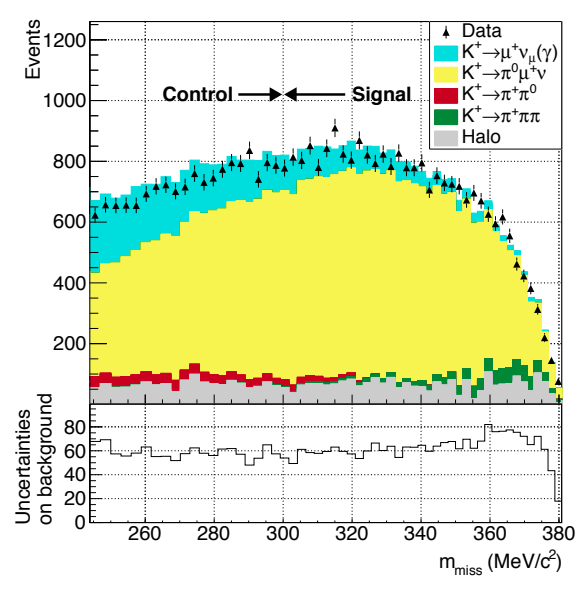

(a) $K^{+} \rightarrow \mu^{+} N 2007$ analysis.

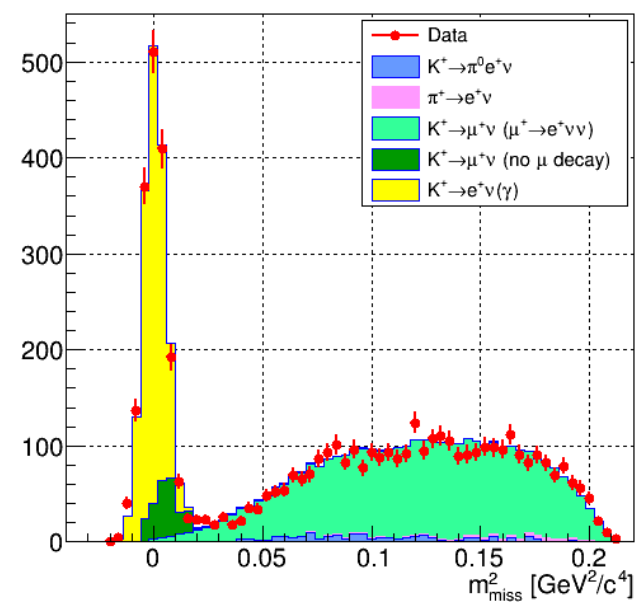

(b) $K^{+} \rightarrow e^{+} N 2015$ analysis.

Figure 3. Missing mass distribution for the selected events in the muon channels together with the MC background estimation (left) and in the positron channel (right).

\subsection{Upper limits}

The peak search was performed scanning the two signal regions with a step of $1 \mathrm{MeV} / \mathrm{c}^{2}$ and in a window of two times the resolution (evaluated with MC simulation) of the HNL mass for the specific bin taken into account. The Rolke-Lopez statistical method was applied to find the $90 \%$ confidence intervals for the number of the reconstructed $\mathrm{K}^{+} \rightarrow \ell^{+} N$ decays. No statistically significant signal was observed in both analysis and therefore only upper limits were set on the production of HNL, figure 4 . In 2015 data a relaxed cut was applied on the longitudinal vertex position for mass hypothesis above $350 \mathrm{MeV} / c^{2}$, which resulted in a discontinuity of the selection acceptance visible in the upper limit of figure 4.

The upper limits on the number of observed signal events were then converted into upper limits on the branching fraction $\mathcal{B R}\left(K^{+} \rightarrow \ell^{+} N\right)$ for each HNL mass hypothesis using the relation between the number of signal events $\left(N_{\text {sig. }}\right)$, the total number of kaon decay $\left(N_{K}\right)$ and the acceptance $(A c c$.): $U L\left(\mathcal{B R}\left(K^{+} \rightarrow \ell^{+} N\right)\right)=\frac{U L\left(N_{\text {sig. }}\right)}{N_{K} \cdot A c c .}$ figure 5 . 


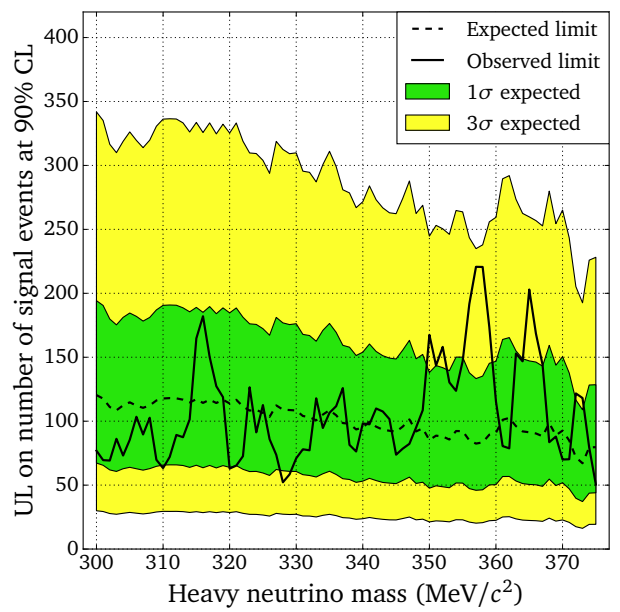

(a) $K^{+} \rightarrow \mu^{+} N 2007$ analysis.

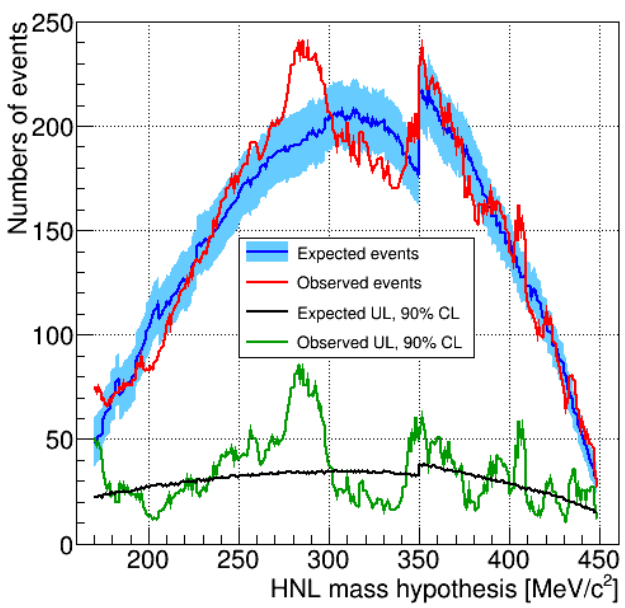

(b) $K^{+} \rightarrow e^{+} N 2015$ analysis.

Figure 4. Expected and observed upper limits on the number of signal events at 90\% CL for each HNL mass hypothesis.

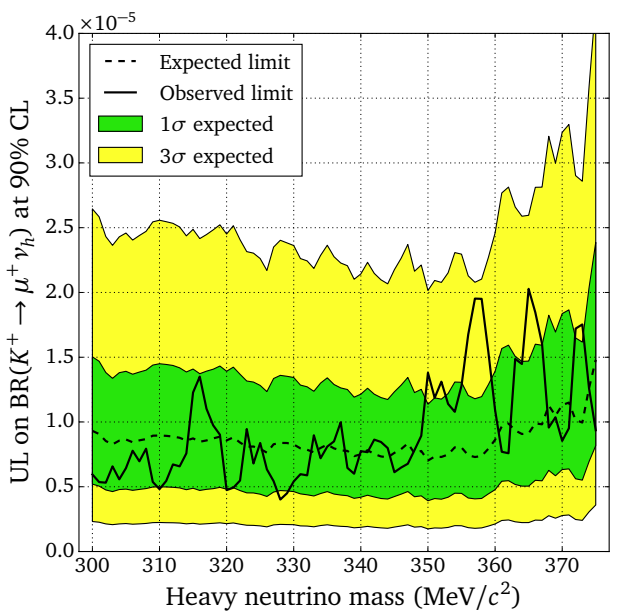

(a) $K^{+} \rightarrow \mu^{+} N 2007$ analysis.

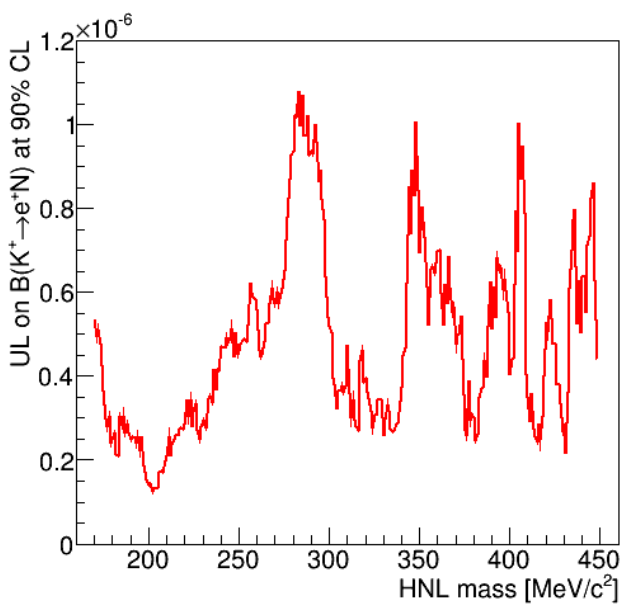

(b) $K^{+} \rightarrow e^{+} N 2015$ analysis.

Figure 5. Expected and observed upper limits on $\mathcal{B R}\left(K^{+} \rightarrow \ell^{+} N\right)$ at $90 \% \mathrm{CL}$ for each HNL mass hypothesis.

\section{Conclusion}

New upper limits are set by NA48/2 and NA62 experiments in the search for HNL both in decay and production.

The upper limit on the branching ratio of the LNV decay $\mathcal{B R}\left(K^{ \pm} \rightarrow \pi^{\mp} \mu^{ \pm} \mu^{ \pm}\right)$is the world best limit. The peak search of HNL in $K^{+} \rightarrow \ell^{+} N$ decays improves the existing limits on both matrix elements 
$U_{\mu 4}$ and $U_{e 4}$ in the analyzed signal region. A comparison with previous HNL searches in $\pi^{+}$([13],[14]) and $K^{+}([15],[16])$ sectors is shown in figure 6 .

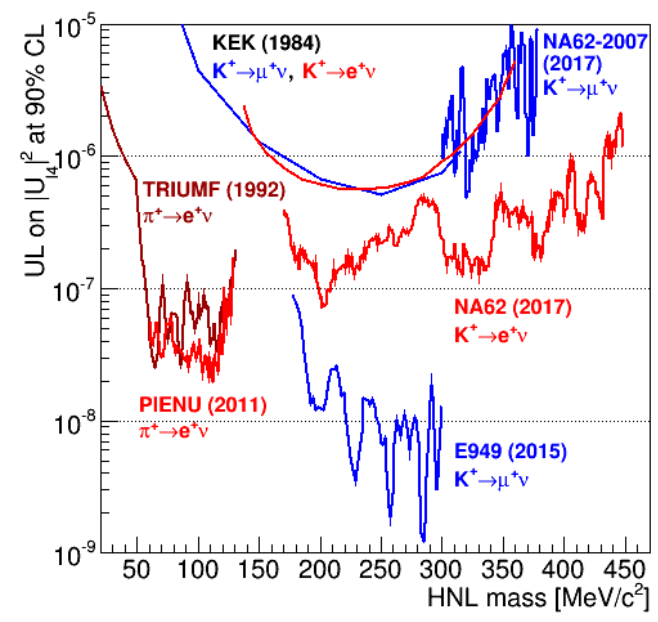

Figure 6. Upper limits on $\left|U_{\ell 4}\right|^{2}$ at $90 \%$ CL from the NA62 analysis compared to the limits established by earlier HNL production searches in $\pi^{+}$and $K^{+}$decays.

\section{References}

[1] T. Asaka and M. Shaposhnikov, Phys. Lett. B620, 17 (2005)

[2] R.E. Shrock, Phys. Lett. B96, 159 (1980)

[3] H. Burkhardt et al., Nucl. Instrum. Meth. A268, 116-143 (1988)

[4] V. Fanti et al. (NA48 Collaboration), Nucl. Instrum. Meth. A574, 433 (2207)

[5] G. Anelli et al. "Proposal to measure the rare decay $K^{+} \rightarrow \pi^{+} v \bar{v}$ at the CERN SPS", CERNSPSC-2005-013, SPSC-P-326 (2005)

[6] C. Lazzeroni et al. (NA62 Collaboration), Phys. Lett. B719, 326 (2013)

[7] E. Cortina Gil et al., JINST 12, P05025 (2017)

[8] A.J. Buras, D. Buttazzo, K.Girrbach-Noe, R. Knegjens, JHEP11, 033 (2015)

[9] J.R. Batley et al. (NA48/2 Collaboration), Eur. Phys. J. C52, 875 (2007)

[10] W.A. Rolke and A.M. Lopez, Nucl. Instrum. Meth. A458, 745 (2001).

[11] J.R. Batley et al. (NA48/2 Collaboration), Phys. Lett. B769, 67 (2017)

[12] C. Lazzeroni et al. (NA62 Collaboration), Phys. Lett. B772, 712 (2017)

[13] D. Britton et al., Phys. Rev. D46, R885 (1992)

[14] A. Aoki et al., Phys. Rev. D84, 052002 (2011)

[15] T. Yamazaki et al., Conf. Proc. C840719, 262 (1984)

[16] A.V. Artamonov et al. (BNL E949 Collaboration), Phys. Rev. D91, 052001 (2015) 